\title{
Mouse hepatitis virus receptor as a determinant of the mouse susceptibility to MHV infection
}

\author{
Fumihiro Taguchi ${ }^{1}{ }^{*}$ and Asuka Hirai-Yuki ${ }^{2}$ \\ ${ }^{1}$ Laboratory of Virology and Viral Infections, Department of Veterinary Medicine, Nippon Veterinary and Life Science University, Musashino, Tokyo, Japan \\ ${ }^{2}$ Laboratory of Animal Care, National Institute of Infectious Disease, Musashi-Murayama, Tokyo, Japan
}

\author{
Edited by: \\ Kazutaka Terahara, National Institute \\ of Infectious Diseases, Japan \\ Reviewed by: \\ Kazutaka Terahara, National Institute \\ of Infectious Diseases, Japan \\ Nicole Beauchemin, McGill \\ University, Canada \\ ${ }^{*}$ Correspondence: \\ Fumihiro Taguchi, Department of \\ Veterinary Medicine, Laboratory of \\ Virology and Viral Infections, Nippon \\ Veterinary and Life Science \\ University, 1-7-1 Kyonan, Musashino, \\ Tokyo 180-8602, Japan. \\ e-mail: ftaguchi@nvlu.ac.jp
}

In this review, we report that the receptor of mouse hepatitis virus (MHV), carcinoembryonic antigen-related cell adhesion molecule 1 (CEACAM1), is an important determinant of mouse susceptibility to MHV infection. This finding was revealed by using mouse strains with two different allelic forms of the MHV receptor, Ceacam1a and Ceacam1b. Although previous studies indicated that susceptibility is determined by a single gene, Ceacam1, our recent work in gene-replaced mice with chimeric Ceacam 1 pointed toward the involvement of other host factors (genes) in the susceptibility. Studies on mouse susceptibility to MHV, as well as the factors involved in their susceptibility, are overviewed.

Keywords: MHV, coronavirus, receptor, MHV receptor, mouse, MHV resistant gene, gene-replacement

\section{INTRODUCTION}

Mouse hepatitis virus (MHV) is a murine coronavirus which causes a wide range of diseases in the mouse and rat, including hepatitis, enteritis, respiratory diseases, and encephalomyelitis in the central nervous system (Wege et al., 1982). There are also great differences in tissue tropism and virulence among various strains and isolates of MHV (Hirano et al., 1981; Wege et al., 1982). Complex factors, such as age and immunological status of the host animal, dose, and route of virus inoculation, affect disease outcomes (Taguchi et al., 1977; Hirano et al., 1981). Naturally occurring cases of MHV infections in mouse colonies are generally enteric infections that are not highly pathogenic to immunocompetent adult mice, while virus infections are mostly of a persistent nature (Ishida et al., 1978; Homberger, 1997). Viruses shed in the feces from persistently infected mice are a source of acute and chronic fatal diseases encountered in mice prone to MHV infection, e.g., diarrhea of suckling mice and wasting syndrome in immunodeficient nude mice (Hirano et al., 1975; Tamura et al., 1977; Ishida et al., 1978). Thus, the asymptomatic infection of adult mice is a major problem in animal facilities where MHV-free mice are used for experiments and evokes serious problems in terms of maintaining animals of sufficient quality to allow scientists to perform experiments with high reproducibility and reliability.

It is well known that there is a difference in susceptibility to MHV infection among mouse strains. A number of works have reported differing susceptibilities to MHV infection among mouse strains from 1960 to the present (Bang and Warwick, 1960; Taguchi et al., 1976; Stohlman and Frelinger, 1978; Knobler et al., 1981). Bang and Warwick (1960) showed that the $\mathrm{C} 3 \mathrm{H}$ inbred strain was resistant to MHV-2 infection, while Princeton outbred mice were deemed susceptible. They further showed that susceptibility was determined by a single gene, expressed in macrophages (Bang and Warwick, 1960). Stohlman and Frelinger (1978) reported, using a neurotropic MHV, JHMV strain, that SJL mice are resistant, while others are susceptible. By cross and backcross studies, they indicated that susceptibility is dominant, as determined by a major gene and a gene marginally influencing its susceptibility (Stohlman and Frelinger, 1978). Thereafter, Smith et al. (1984) identified the locus of the gene that determines the susceptibility of mice to MHV infection to be on chromosome 7. From those findings, it was postulated that mouse susceptibility to MHV infection is dominant and determined mostly by a single gene located on chromosome 7. On the basis of those findings, Holmes and her colleagues identified an MHV receptor.

\section{IDENTIFICATION OF MHV RECEPTOR}

Holmes and co-workers tried to find a receptor protein by using susceptible BALB/c and resistant SJL/J mice (Boyle et al., 1987). They prepared cell membrane fractions from MHV target tissues, intestines, and liver, of those mice and showed, by using a viral overlay protein blot assay (whereby virions bind to the protein on a membrane filter prepared by Western blotting), that BALB/c mice express a molecule of $110-120 \mathrm{kDa}$ on cell membranes to bind MHV virus particles. However, in the same fraction of SJL mice, they failed to find the protein that would bind MHV (Boyle et al., 1987; Williams et al., 1990). Accordingly, they speculated that the protein was a receptor for MHV and determines mouse susceptibility. Thereafter, they isolated the protein and analyzed the partial amino acid sequence of the protein, which suggested to them that the protein is similar to a carcinoembryonic antigen, CEA (Williams et al., 1991). They finally isolated a cDNA clone and identified the protein to be a biliary glycoprotein of the CEA family (Dveksler et al., 1991), now called carcinoembryonic antigen-related cell adhesion molecule 1 (CEACAM1; Beauchemin et al., 1999). The expression of this protein in non-permissive cells converted them into cells that were permissive to MHV infection, 
a finding that indicated this protein serves as an MHV receptor (Dveksler et al., 1991). These findings suggested to them that the susceptibility of the mouse to MHV infection is determined by the presence or absence of this molecule. Soon after these findings were published, they reported that the CEACAM1 counterpart is expressed in the SJL (Dveksler et al., 1993b); however, they also reported that the CEACAM1 counterpart found in SJL is also functional as a receptor for $\mathrm{MHV}$ when expressed in non-permissive cells, although the receptor functionality is less efficient compared with the CEACAM1 found in BALB/c (Dveksler et al., 1993b). Holmes and co-workers explained that the relatively small difference in receptor function between BALB/c CEACAM1 and SJL CEACAM1 could result in the very large biological differences in the multiple cycle infections that are required to cause disease in animals (Dveksler et al., 1993b). There is a slight difference in amino acid sequences between CEACAM1 found in most mouse strains and CEACAM1 expressed in SJL in the MHV-binding region (Rao et al., 1997; Beauchemin et al., 1999); these are allelic forms and called CEACAM1a for BALB/c type and CEACAM1b for SJL type (Beauchemin et al., 1999). In contrast to the hypothesis by Holmes and co-workers, Yokomori and Lai (1992) expressed CEACAM1a derived from C57BL/6 and CEACAM1b from SJL mice in MHV-non-permissive cells and found no significant difference in viral growth between cells expressing CEACAM1a and those with CEACAM1b, which may mean that the susceptibility difference observed between those two mouse strains may not be attributable to MHV receptor protein.

\section{STRUCTURE AND MHV-BINDING ACTIVITY OF CEACAM1s}

There are at least four different types of CEACAM1 isoforms derived by alternative splicing (Beauchemin et al., 1999). CEACAM1 belongs to an immunoglobulin ( Ig) superfamily and is composed of an ectodomain, transmembrane domain (TM), and cytoplasmic tail (Cy). The ectodomain consists of four Ig constant region-like domains, i.e., the $\mathrm{N}, \mathrm{A} 1, \mathrm{~B}$, and $\mathrm{A} 2$ domains from the $\mathrm{N}$ terminus of the molecule (Dveksler et al., 1991; Beauchemin et al., 1999). Alternative splicing generates the molecules with two ectodomains, composed of $\mathrm{N}$ and $\mathrm{A} 2$, while those four- and twodomain forms have either long or short $\mathrm{Cy}$, which results in the four different forms of CEACAM1 (Beauchemin et al., 1999). Also as described above, there are two allelic forms in CEACAM1, 1a, and $1 \mathrm{~b}$, and, thus, at least eight totally different $\mathrm{MHV}$ receptor molecules exist (Beauchemin et al., 1999). Recently, some CEACAM1 molecules deleting TM and Cy were found in intestinal epithelium and intestinal secretions, and those neutralize MHV (Terahara et al., 2009).

The molecule on MHV virion that binds to the receptor is the spike (S) protein with ca. 180-200 kDa class I fusion glycoprotein (Bosch et al., 2003). One spike is composed of a trimer of the S protein. The S protein of most MHV is cleaved in the middle of the molecule, and the $\mathrm{N}$ terminal subunit is called the $\mathrm{S} 1$ and $\mathrm{C}$ terminal subunits S2, respectively (Sturman et al., 1985). N terminal 330 amino acids in $\mathrm{S} 1$ are responsible for receptor binding (Kubo et al., 1994), and S2 is critical for envelope-cell membrane fusion, namely, cell entry (Bosch et al., 2003).

The $\mathrm{N}$ domain of CEACAM1 alone is sufficient to bind MHV; a soluble form consisting of the $\mathrm{N}$ domain alone works efficiently to bind to MHV (Dveksler et al., 1993a; Miura et al., 2004). Also, the
$\mathrm{N}$ domain alone can neutralize viruses and induce conformational change in the $\mathrm{S}$ protein. Moreover, the binding of the $\mathrm{N}$ domain to the $S$ protein of MHV induces the fusion activation of the $S$ protein (Taguchi and Matsuyama, 2002; Miura et al., 2004). However, when the $\mathrm{N}$ domain alone is expressed on the membrane (which has both TM and Cy), this molecule fails to work as a functional receptor (Dveksler et al., 1993a; Miura et al., 2004). This may be due to its short molecule that is buried among various cell surface molecules and thus fails to have a chance to attach to the virions (Dveksler et al., 1993a). We have compared the virus-binding activity of CEACAM1a and CEACAM1b. Soluble CEACAM1a and CEACAM1b composed of $\mathrm{N}$ and $\mathrm{A} 2$ domains prepared on nitrocellulose paper were allowed to attach to an MHV virion (viral protein blot assay). By this method, CEACAM1a binds to virions more than 300-fold efficiently when compared with CEACAM1b (Ohtsuka et al., 1996). We also examined the neutralization activity of the two soluble proteins, finding that, in this assay, CEACAM1a is highly reactive and neutralizes MHV more than 300-fold efficiently than does CEACAM1b. These results are in agreement with the observations of the Holmes lab (Boyle et al., 1987). However, when those proteins are expressed in MHV-non-permissive BHK cells, CEACAM1a exhibits only a 10- to 30-fold higher receptor function when compared with that of CEACAM1b, and, again, this finding is similar to those obtained by the Holmes lab (Boyle et al., 1987). Accordingly, from these findings, we concluded that the small difference in receptor function between CEACAM1a and CEACAM1b will be amplified after several viral growths, as also postulated by Holmes and her collaborators. As for the receptor binding site in the N domain of CEACAM1, Gallagher and coworkers reported six contiguous amino acids from 38 to 43 in the $\mathrm{N}$ domain different between CEACAM1a and CEACAM1b play an important role in the differences seen in receptor function (Rao et al., 1997). Others showed that amino acids 34-52 are involved in virus-binding (Wessner et al., 1998).

\section{ANALYSIS OF THE ROLE OF CEACAM1 IN THE MHV SUSCEPTIBILITY OR RESISTANCE OF MICE}

The above data suggested to us that CEACAM1 is an important factor to determine the susceptibility of mice and could be a product of the gene responsible for susceptibility mapped in a locus on chromosome 7 by Smith et al. (1984). This idea is strengthened by the fact that the MHV receptor Ceacam1 gene is mapped to the same region on chromosome 7 (Robbins et al., 1991). If the CEACAM1 receptor is a determinant of susceptibility, and susceptibility is dominant over resistance, then mice with CEACAM1a/CEACAM1a and CEACAM1a/CEACAM1b are susceptible and only mice with a CEACAM1b/CEACAM1b phenotype, such as SJL mice, are resistant. To assess this possibility, we have mated BALB/c and SJL to generate F1 mice, and then we obtained mice backcrossed to SJL. We also produced F2 mice from BALB/c and SJL mice. By using these mice, we examined the relationship between the mouse genotype of Ceacam 1 and its susceptibility to MHV. Of more than 120 backcrossed and F2 mice, those with Ceacam1a/Ceacam1a and Ceacam1a/Ceacam1b were fully susceptible, while those with Ceacam $1 b / C e a c a m 1 b$ were resistant (Ohtsuka and Taguchi, 1997). These experimental results are well in accordance with the hypothesis, i.e., that mouse susceptibility to MHV is determined by a receptor gene. We have also 
studied the relationship between MHV susceptibility and CEACAM1 allelic forms by using wild mice (Ohtsuka et al., 2001). The study showed that most of subspecies of wild mice distributed worldwide express both or either CEACAM1a and CEACAM1b. Among those mice, CEACAM1a-expressing ones showed high susceptibility to MHV, while those with CEACAM1b were not highly susceptible, being in good agreement to the result and hypothesis obtained by mouse cross and backcross experiment shown above.

It has been reported in studies using gene-knockout mice that CEACAM1a is a critical factor for mouse susceptibility to MHV infection (Blau et al., 2001; Hemmila et al., 2004). Beauchemin and co-workers produced mice whose Ceacam 1 gene is partially disrupted or mice in which the Ceacamla gene was deleted and showed that those mice showed a reduced susceptibility and no susceptibility to MHV infection, respectively (Blau et al., 2001; Hemmila et al., 2004). These findings clearly show that CEACAM1 is an important factor in determining the susceptibility to MHV. However, these studies did not explain the difference of susceptibility to MHV infection observed between BALB/c with CEACAM1a and SJL with CEACAM1b receptor protein.

\section{ANALYSIS OF GENE-REPLACED MICE}

If the differing susceptibilities between CEACAM1a-expressing mice and SJL with CEACAM1b are determined by CEACAM1, then MHV-susceptible mice with CEACAM1a are converted to SJL type-resistant mice, when the Ceacam1a mouse gene is replaced with a Ceacam1b gene. We have produced C57BL/6 (B6) mice whose original Ceacam1a gene is replaced by a Ceacam $1 b$ gene (Hirai et al., 2010). Since it has been revealed that the chimeric CEACAM1a, which is replaced with 1-70 amino acids of the $\mathrm{N}$ terminus of $\mathrm{N}$ domain with CEACAM1b counterpart, functioned as CEACAM1b, suggesting that there is a critical region in 1-70 amino acids to determine the difference of receptor functionality between CEACAM1a and CEACAM1b (Wessner et al., 1998). Thus, we produced $\mathrm{B} 6$ mice whose $\mathrm{N}$ terminal region (1-70 amino acids) is replaced by CEACAM1b (we call it CEACAM1ba) to see the mouse susceptibility is determined by CEACAM1. CEACAM1ba has two N-linked glycosylation sites in the $\mathrm{N}$ domain as CEACM1b, while CEACAM1a has three sites, showing that chimeric CEACAM1ba is more like CEACAM1b rather than CEACAM1a. Those gene-replaced mice expressed the chimeric protein in the tissues or cells where CEACAM1a and CEACAM1b were expressed in B6 and SJL mice, respectively. It was also revealed that there is no significant difference in the expression level of CEACACM1a and CEACAM1ba in B6 and chimeric B6 mice (Hirai et al., 2010). Additionally, the chimeric CEACAM1 did not react with the CEACAM1a-specific monoclonal antibody, CC1 as reported previously (Wessner et al., 1998). We then examined the susceptibility of mice having a chimeric Ceacam1. The gene-replaced mice, when compared to B6 mice, were resistant to a lethal dose of MHV-A59 infection, a finding similar to those in SJL mice that were resistant to the infection. However, when we

\section{REFERENCES}

Bang, F. B., and Warwick, A. (1960). Mouse macrophages as host cells for the mouse hepatitis virus and the genetic basis of their susceptibility.

examined virus growth in B6, SJL, and the gene-replaced mice, high titer of MHV were detected in the liver and other target tissues of B6 mice, while low levels of virus titer were recorded in SJL mice, findings which are similar to the results we have thus far obtained. Interestingly, no virus growth was detected in the mice having gene-replacement with chimeric CEACAM1, indicating the gene-replaced mice showed a much higher resistance to MHV infection than did the SJL mice. Since the virus-binding domain is derived from SJL, the chimeric mouse susceptibility should be similar to that in SJL mice, if our hypothesis is correct. However, at the moment, we have no convincing explanation on the difference in susceptibility of SJL and chimeric mice with identical MHV-binding site on CEACAM1.

Two other molecules, CEACAM2 (Nedellec et al., 1994) and pregnancy-specific glycoprotein (PSG; Chen et al., 1995), have so far been reported to work as MHV receptor. These proteins, however, seem unlikely to function as an MHV receptor in the mouse, since CEACAM1 knockout mice produced in Beauchemin lab showed complete resistance to MHV-A59 infection (Hemmila et al., 2004),. Moreover, as there are no substantial differences in amino acid sequences in those two receptor proteins expressed in B6 and SJL, there is little possibility that those proteins are involved in the differences of MHV susceptibility between SJL and chimeric mice. One point to be further studied is the effect of FVB Cre mouse that is used to exclude neomycin resistant gene from initially produced chimeric mouse (Hirai et al., 2010). To exclude the FVB mouse genes, we performed the backcross of neomycin deleted mouse to B6 mouse by 11 generations, however, some FVB mouse gene still remaining in backcrossed mice could have some unexpected effects on the chimeric mice. It is important to study whether any of FVB mouse genes are involved in the high resistance of chimeric mice.

We did observe that peritoneal macrophages from chimeric mice, which express chimeric CEACAM1ba, were resistant to $\mathrm{MHV}$, while chimeric CEACAM1 expressed in cultured cell lines, such as BHK, showed MHV susceptibility, slightly lower than that in cells expressing CEACAM1b. From these findings, it could be postulated that some cellular factor(s) other than CEACAM1 could modify animal susceptibility. This idea appears to be in accordance with findings by Stohlman and Frelinger (1978), who postulated two factors to determine mouse strain differences in susceptibility to MHV infection. Studies to elucidate such factors are in progress.

\section{ACKNOWLEDGMENTS}

We thank Nobuhisa Ohtsuka, Toshio Ikeda, Tateki Kikuchi, and other members in National Institute of Neuroscience NCNP and colleagues in National Institute of Infectious Diseases, where our works shown in this review have been performed. Those works were financially supported by the grants-in-aid from Ministry of Education, Culture, Sports, Science and Technology of Japan.

Karlsson, A., Kuroki, M., Lin, S. H., Lucka, L., Najjar, S. M., Neumaier, M., Obrink, B., Shively, J. E., Skubitz, K. M., Stanners, C. P., Thomas, P., Thompson, J. A., Virji, M., von
Kleist, S., Wagener, C., Watt, S., and Zimmermann, W. (1999). Redefined nomenclature for members of the carcinoembryonic antigen family. Exp. Cell Res. 252, 243-249. 
Blau, D. M., Turbide, C., Tremblay, M., Olson, M. A., Letourneau, S., Michaliszyn, E., Jothy, S., Holmes, K. V., and Beauchemin, N. (2001). Targeted disruption of the Ceacam 1 (MHVR) gene leads to reduced susceptibility of mice to mouse hepatitis virus infection. J. Virol. 75, 8173-8186.

Bosch, B. J., van der Zee, R., de Haan, C. A. M., and Rottier, P. J. M. (2003). The coronavirus spike protein is a class I fusion protein: structural and functional characterization of the fusion core complex. J. Virol. 77, 8801-8811.

Boyle, J. F., Weismiller, D. G., and Holmes, K. V. (1987). Genetic resistance to mouse hepatitis virus correlates with absence of virus-binding activity on target tissues. J. Virol. 61, 185-189.

Chen, D. S., Asanaka, M., Yokomori, K., Wang, F., Hwang, S. B., Li, H., and Lai, M. M. C. (1995). A pregnancyspecific glycoprotein is expressed in the brain and serve as a receptor for mouse hepatitis virus. Proc. Natl. Acad. Sci. U.S.A. 92, 12095-12099.

Dveksler, G. S., Pensiero, M. N., Cardellichio, C. B., Williams, R. K., Jiang, G. S., Holmes, K. V., and Dieffenbach, C. W. (1991). Cloning of the mouse hepatitis virus (MHV) receptor: expression in human and hamster cell lines confers susceptibility to MHV. J. Virol. 65, 6881-68891.

Dveksler, G. S., Pensiero, M. N., Dieffenbach, C. W., Cardellichio, C. B., Basile, A. A., Elia, P. E., and Holmes, K. V. (1993a). Mouse hepatitis virus strain A59 and blocking antireceptor monoclonal antibody bind to the $\mathrm{N}$ terminal domain of cellular receptor. Proc. Natl. Acad. Sci. U.S.A. 90, 1716-1720.

Dveksler, G. S., Dieffenbach, C. W., Cardellichio, C. B., McCuaig, K., Pensiero, M. N., Jiang, G. S., Beauchemin, N., and Holmes, K. V. (1993b). Several members of the mouse carcinoembryonic antigenrelated glycoprotein family are functional receptors of the coronavirus mouse hepatitis virus-A59. J. Virol. 67, 1-8.

Hemmila, E., Turbide C Olson, M., Jothy, S., Holmes, K. V., and Beauchemin, N. (2004). Ceacamla-/mice are completely resistant to infection by murine coronavirus mouse hepatitis virus A59. J. Virol. 78, 10156-10165.

Hirai, A., Ohtsuka, B., Ikeda, T., Taniguchi, R., Blau, D., Nakagaki, K., Miura, H. S., Ami, Y., Yamada,
Y. K., Itohara, S., Holmes, K. V., and Taguchi, F. (2010). Role of mouse hepatitis virus (MHV) receptor murine CEACAM1 in the resistance of mice to MHV infection: studies of mice with chimeric mCEACAM1a and mCEACM1b. J. Virol. 84, 6654-6666.

Hirano, N., Murakami, T., Taguchi, F., Fujiwara, K., and Matumoto, M. (1981). Comparison of mouse hepatitis virus strains for pathogenicity in weanling mice infected by various route. Arch. Virol. 70, 69-73.

Hirano, N., Tamura, T., Taguchi, F., Ueda, K., and Fujiwara, K. (1975). Isolation of low-virulent mouse hepatitis virus from nude mice with wasting syndrome and hepatitis. Jpn. J. Exp. Med. 45, 429-432.

Homberger, F. R. (1997). Enterotropic mouse hepatitis virus. Lab. Anim. 31, 97-115.

Ishida, T., Taguchi, F., Lee, Y., Yamada, A., Tamura, T., and Fujiwara, K. (1978). Isolation of mouse hepatitis virus from infant mice with fatal diarrhea. Lab. Anim. Sci. 28, 269-276.

Knobler, R. L., Haspel, M. V., and Oldstone, M. B. A. (1981). Mouse hepatitis virus type 4 (JHM strain)induced fatal central nervous system disease. I. Genetic control and the murine neuron as the susceptible site of disease. J. Exp. Med. 153, 832-843.

Kubo, H., Yamada, Y. K., and Taguchi, F. (1994). Localization of neutralizing epitopes and the receptor-binding site within the amino-terminal 330 amino acids of the murine coronavirus spike protein. J. Virol. 68 , 5403-5410.

Miura, S. H., Nakagaki, K., and Taguchi, F. (2004). $\mathrm{N}$ terminal domain of murine coronavirus receptor CEACAM1 is responsible for fusogenic activation and conformational changes of the spike protein. J. Virol. 78, 216-223.

Nedellec, P., Dveksler, G. S., Danieles, E., Turbide, C., Chow, B., Basile, A. A., Holmes, K. V., and Beauchemin, N. (1994). Bgp-2, a new member of the carcinoembryonic antigenrelated gene family, encodes an alternative receptor for mouse hepatitis viruses. J. Virol. 68, 4525-4537.

Ohtsuka, N., and Taguchi, F. (1997). Mouse susceptibility to mouse hepatitis virus infection is linked to viral receptor genotype. J. Virol. 71, 8860-8863.

Ohtsuka, N., Tsuchiya, K., Honda, E., and Taguchi, F. (2001). A study on mouse hepatitis virus receptor genotype in the wild mouse. $A d v$. Exp. Med. Biol. 494, 237-240.

Ohtsuka, N., Yamada, Y. K., and Taguchi, F. (1996). Difference in virus-binding activity of two distinct receptor proteins for mouse hepatitis virus. J. Gen. Virol. 77, 1683-1692.

Rao, P. V., Kumari, S., and Gallagher, T. M. (1997). Identification of a contiguous 6-residue determinant in the MHV receptor that controls the level of virion binding to cells. Virology 229, 336-348.

Robbins, J., Robbins, P., Kozak, C. A., and Callahan, R. (1991). The mouse biliary glycoprotein gene (Bgp): partial nucleotide sequence, expression and chromosomal assignment. Genomics 10, 583-587.

Smith, M. S., Click, R. E., and Plagemann, P. G. W. (1984). Control of mouse hepatitis virus replication in macrophages by a recessive gene on chromosome 7. J. Immunol. 134 428-432.

Stohlman, S. A., and Frelinger, J. A. (1978). Resistance to fatal central nervous system disease by mouse hepatitis virus, strain JHM. 1. Genetic analysis. Immunogenetics 6 , 277-281.

Sturman, L. S., Ricard, C. S., and Holmes, K. V. (1985). Proteolytic cleavage of the E2 glycoprotein of murine coronavirus: activation of cell fusing activity of virions by trypsin and separation of two different $90 \mathrm{~K}$ cleavage fragments. J. Virol. 56, 904-911.

Taguchi, F., Aiuchi, M., and Fujiwara, K. (1977). Age-dependent response of mice to a mouse hepatitis virus, MHV-S. Jpn. J. Exp. Med. 47, 109-115.

Taguchi, F., Hirano, N., Kiuchi, Y., and Fujiwara, K. (1976). Difference in response of mouse hepatitis virus among susceptible mouse strains. Jpn. J. Microbiol. 20, 293-302.

Taguchi, F., and Matsuyama, S. (2002). Soluble receptor potentiates receptor-independent infection by murine coronavirus. J. Virol. 76 , 950-958.

Tamura, T., Taguchi, F., Ueda, K., and Fujiwara, K. (1977). Persistent infection with mouse hepatitis virus of low virulence in nude mice. Microbiol. Immunol. 21, 683-691.

Terahara, K., Yoshida, M., Taguchi, F., Igarashi, O., Nochi, T., Gotoh, Y., Yamamoto, T., TsunetsuguYokota, Y., Beauchemin, N., and Kiyono, H. (2009). Expression of newly identified secretory CEACAM1a isoforms in the intestinal epithelium. Biochem. Biophys. Res. Commun. 383, 340-346.

Wege, H., Siddell, S. G., and ter Meulen, V. (1982). The biology and pathogenesis of coronaviruses. Curr. Top. Microbiol. Immunol. 99 , 165-200.

Wessner, D. R., Shick, P. C., Lu, J. H., Cardellichio, C. B., Gagneten, S. E., Beauchemin, N., Holmes, K. V., and Dveksler, G. S. (1998). Mutational analysis of the virus and monoclonal antibody binding sites in MHVR, the cellular receptor of the murine coronavirus mouse hepatitis virus strain A59. J. Virol. 72, 1941-1948.

Williams, R. K., Jiang, G., Synder, S. W., Frana, M. F., and Holmes, K. V. (1990). Purification of the 110kilodalton glycoprotein receptor for mouse hepatitis virus (MHV)-A59 from mouse liver and identification of a nonfunctional, homologous protein in MHV-resistant SJL/J mice. J. Virol. 64, 3817-3823.

Williams, R. K., Jiang, G. S., and Holmes, K. V. (1991). Receptor for mouse hepatitis virus is a member of the carcinoembryonic antigen family of glycoproteins. Proc. Natl. Acad. Sci. U.S.A. 88, 5533-5536.

Yokomori, K., and Lai, M. M. C. (1992). The receptor for mouse hepatitis virus in the resistant mouse strain SJL is functional: implications for the requirement of a second factor for viral infection. J. Virol. 66, 6931-6938.

Conflict of Interest Statement: The authors declare that the research was conducted in the absence of any commercial or financial relationships that could be construed as a potential conflict of interest.

Received: 22 December 2011; accepted: 09 February 2012; published online: 24 February 2012.

Citation: Taguchi F and Hirai-Yuki A (2012) Mouse hepatitis virus receptor as a determinant of the mouse susceptibility to MHV infection. Front. Microbio. 3:68. doi: 10.3389/fmicb.2012.00068

This article was submitted to Frontiers in Virology, a specialty of Frontiers in Microbiology.

Copyright () 2012 Taguchi and HiraiYuki. This is an open-access article distributed under the terms of the Creative Commons Attribution Non Commercial License, which permits noncommercial use, distribution, and reproduction in other forums, provided the original authors and source are credited. 